\title{
MODIFICATION OF THE RELEASE OF DIPYRIDAMOLE FROM CERTAIN POLYMERIC SYSTEMS
}

\author{
Ahmed E. Aboutaleb, Ali A. Abdel-Rhaman, Mahrous O. Ahmed*, Amany A. Abdel-Rheem \\ Department of Industrial Pharmacy, Faculty of Pharmacy, Assiut University, Assiut, Egypt
}

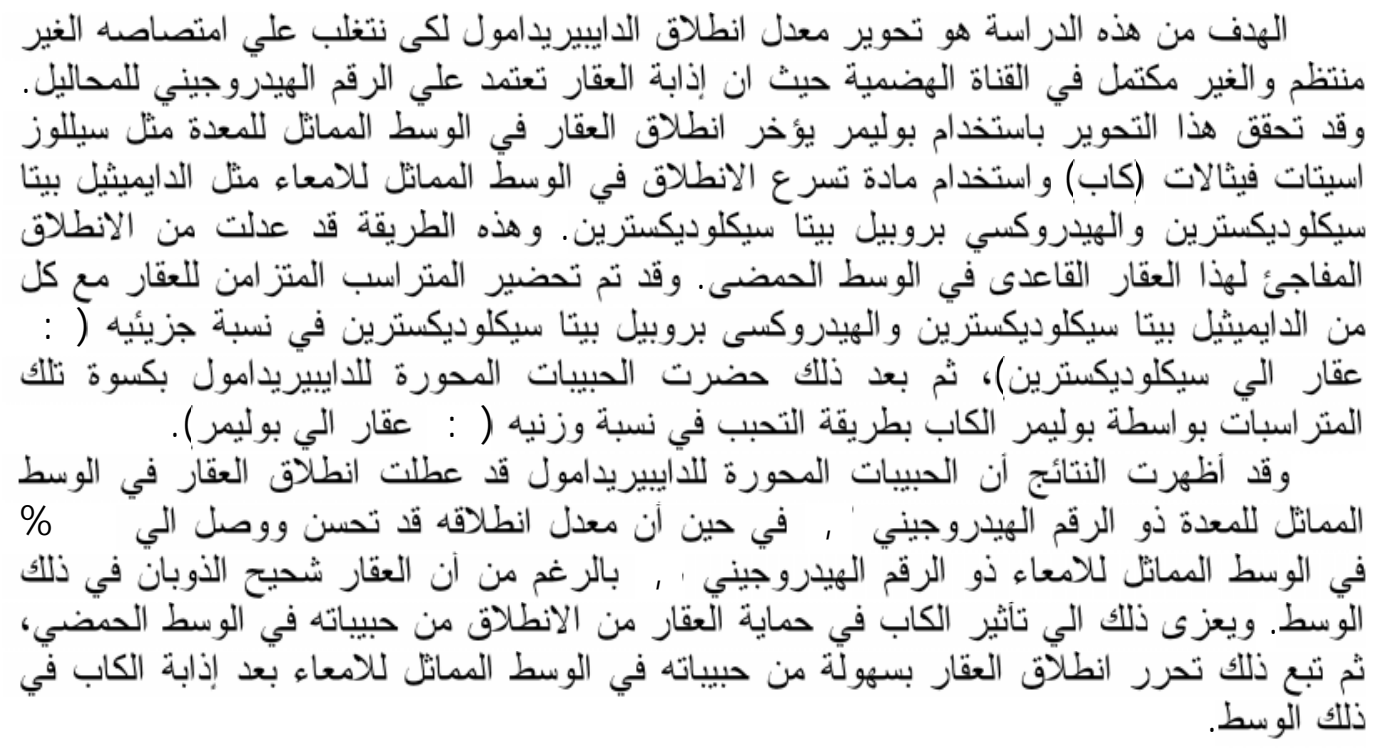

The objective of the present study is to modify the release of dipyridamole (DIP); which has a $\mathrm{pH}$ dependant solubility in order to overcome its irregular and incomplete absorption from the gastrointestinal tract. This was achieved by incorporation of a dissolution retarder such as cellulose acetate phthalate CAP and a dissolution enhancer such as cyclodextrins; (dimethyl beta cyclodextrin DM- $\beta C D$ and hydroxypropyl betacyclodextrin HP- $\beta C D$ ). This was an effective mean of moderating the drastic dissolution behaviour of that basic drug at acidic $p H$. Coevaporates of DIP with DM- $\beta C D$ and DIP with HP- $\beta C D$ in 1:2 molar ratio were prepared, followed by coating of these coevaporates by an enteric polymer (cellulose acetate phthalate, CAP) in 1: 3 drug : polymer weight ratio. In vitro release study was performed for capsules containing the modified granules at $\mathrm{pH}$ values of 1.3, 5 and 6.8. It was found that the drug release from the modified granules was decreased in a simulated gastric fluid and was enhanced in a simulated intestinal fluid and reached up to 100\%. This could be attributed to the protecting effect of CAP for the drug granules in the acidic medium, followed by dissolving of $C A P$ coat in the intestinal fluids which lead the drug to be released easily from the granules.

\section{INTRODUCTION}

Basic drugs having a pH-dependent solubility as dipyridamole (DIP) has bioavailability problems, because, after oral administration, the different $\mathrm{pH}$ values of the gastrointestinal tract result in a drastic change in drug solubility, as DIP is soluble in acidic media and practically insoluble in $\mathrm{pH}$ higher than $5 .{ }^{1,2}$
It may, then, be necessary to increase the drug release as the dosage form progresses through the intestine, so as to compensate the decrease in absorption which generally occurs in the colon with the conventional sustained release dosage forms. ${ }^{3}$

Coprecipitate systems have been extensively used as a mean of enhancing the dissolution of poorly water soluble drugs using water soluble carriers, ${ }^{4-6}$ or alternatively to

Received in 8/1/2004 \& Accepted in 30/3/2004 
control the release of drugs by the same technique, but using insoluble materials. ${ }^{7}$

Cyclodextrins have the ability to entrap drug molecules of appropriate size and polarity in their cavity to form non covalent inclusion compounds. ${ }^{8}$ This may lead to useful modifications of the physical and chemical properties of the guest molecules, allowing the improvement of stability, increasing solubility of poorly water soluble drugs, enhancement of dissolution rate, improvement of membrane permeability and assisting of drugs bioavailability. ${ }^{10,11}$

The aim of this study is to prepare the inclusion complexes of dipyridamole with either DM- $\beta C D$ or HP- $\beta C D$ by coevaporation method. In addition, modified granules of dipyridamole were prepared by granulation of its coevaporates with the above mentioned CDs, using an enteric coating polymer (cellulose acetate phthalate, CAP) to prevent the drug release in the acidic $\mathrm{pH}$ of stomach. As the modified granules of the drug reach the intestine of $\mathrm{pH} 6.8$, the enteric coat of CAP will dissolve leaving the drug to be released easily from its inclusion complex.

\section{EXPERIMENTAL}

\section{Materials}

Dipyridamole was obtained from Chemical Industries Development Company (CID), Cairo, Egypt.

Dimethyl beta cyclodextrin (DM- $\beta C D$ ) was obtained from Toshin Chemicals, CO. Japan.

2-Hydroxypropyl beta cyclodextrin (HP$\beta C D)$ with molar substitution of 0.6 was obtained from Pharmatec, INC, Alachua, Florida, U.S.A.

Cellulose acetate phthalate (CAP) was obtained from Estman Chemical products, INC., U.S.A.

The solvents and the buffer components were of analytical grade. Deionized distilled water was used throughout this study.

\section{Equipment}

USP dissolution apparatus, Hanson Researches Corporation Chatsworth, California, USA. UV-VIS Spectrophotometer, JAS CO., Japan. Differential Scanning Calorimeter, DSC-50I, Shimadzu, Japan.
X-Ray Diffractometer, Philips, PW1710 X-ray, Netherland.

\section{Methods}

\section{1- Preparation of coevaporates}

Coevaporates of DIP with DM- $\beta C D$ and HP- $\beta C D$ were prepare in 1:2 molar ratio using the solvent evaporartion technique. An appropriate amount of $\mathrm{CD}$ was dissolved in ethanol and DIP was dissolved in minimum amount of ethanol and added to the CD solution while stirring. The solvent was allowed to evaporate at room temperature till a dough mass was obtained. The mass was passed through a sieve of $600 \mu \mathrm{m}$ and dried at $37^{\circ}$ for $24 \mathrm{~h}$. Complete drying was attained by obtaining a constant weight and the samples were kept in a dessicator over calcium chloride till used.

\section{2- Preparation of the modified granules}

The dried coevaporates of DIP and CDs were granulated by a solution of the CAP which was prepared by dissolving CAP and drug in 1:3 weight ratio in a mixture of acetone and isopropanol (1:1 volume ratio). The wet mass was sieved through $1600 \mathrm{~m}$ mesh screen to obtain granules which were dried in an oven at $40^{\circ}$. After a proper drying, the granules were resieved through $1000 \mathrm{~m}$ mesh screen. The produced granules were kept in a dessicator over calcium chloride till used.

\section{3- Differential scanning calorimetric investi-gation of the prepared samples (DSC)}

The DSC thermograms were obtained at a scanning rate of $10^{\circ} / \mathrm{min}$ and $\mathrm{N}_{2}$ purge at 40 $\mathrm{ml} / \mathrm{min}$. The instrument was calibrated with an indium standard and the samples were examined in the temperature range $20-300^{\circ}$. The weight sample is $2-5 \mathrm{mg}$ using aluminium pans.

\section{4- X-ray diffraction for the prepared samples (XRD)}

Powder X-ray diffractometry was carried out under the conditions of $\mathrm{CU} / \mathrm{K} \alpha$ radiation (40 Kv, $30 \mathrm{MA}$, Slite 1.5) over 4- $602 \theta$ range.

\section{5- Release studies for the prepared samples \\ The USP XXIII dissolution test apparatus}

2 (paddle type) was used at $100 \mathrm{rpm}$ and 
temperature of $37^{\circ}$. Capsules containing the modified granules with equivalent amounts of $25 \mathrm{mg}$ of dipyridamole were tested. Dissolution media of $\mathrm{pH} 1.3,5$ and 6.8 buffer solutions were used. At appropirate time intervals, $5 \mathrm{ml}$ aliquots were withdrawn, filtered, and analysed spectrophotometrically at $288 \mathrm{~nm}$ for dipyridamole. After each sampling, $5 \mathrm{ml}$ of the same buffer, maintained at $37^{\circ}$, was added to keep the dissolution volume constant. Three replicates were performed for each sample of the modified granules and the average drug release was calculated within 8 hours.

\section{RESULTS AND DISCUSSION}

The physico-chemical properties of the modified granules of DIP were investigated using DSC and X-ray diffraction methods.

\section{Differential scanning calorimetry (DSC)}

Figure (1) shows the DSC thermograms of the pure DIP, the coevaporate with DM- $\beta C D$ in 1:2 molar ratio, the modified granules and granules of DIP with CAP in 1:3 weight ratio. Pure DIP exhibits a sharp endothermic peak at $168.2^{\circ}$ (curve A), corresponding to the melting point of DIP. This indicates that the latter is in its pure crystalline form. Curve B shows the DSC thermogram of the coevaporate of DIP with DM- $\beta C D$ (1:2) molar ratio which shows very large reduction of the endothermic peak of DIP at $168.2^{\circ}$. This indicates that DIP was largely transformed into the amorphous state due to the interaction with $\mathrm{DM}-\beta \mathrm{CD}$. $^{12}$ The modified granules showed complete disappearence of the endothermic peak of DIP, as shown in curve $\mathrm{C}$, which indicates that DIP became amorphous upon coating with CAP. Granules of the drug with CAP show no endothermic peak of the drug which indicates complete coating of DIP by CAP so the drug became completely amorphous (curve $\mathrm{D}$ ).

Figure (2) shows the DSC thermograms of DIP alone, its coevaporate with HP- $\beta$ CD in 1:2 molar ratio and the modified granules containing HP- $\beta C D$ and CAP. The DSC thermogram of the coevaporate (curve B) shows a reduction in the endothermic peak of DIP which may be attributed to the reduction in drug crystallinity. Whereas the modified granules of DIP shows complete disappearance of the endothermic peak of DIP (curve C).
Moreover, DSC curve D shows also disappearance of endothermic peak of DIP in case of its granules with CAP, which could be attributed to complete coating of drug particles by CAP. This assumption is confirmed by appearance of halo diffraction pattern in the $\mathrm{X}$ ray diffractogram of modified granules of DIP.

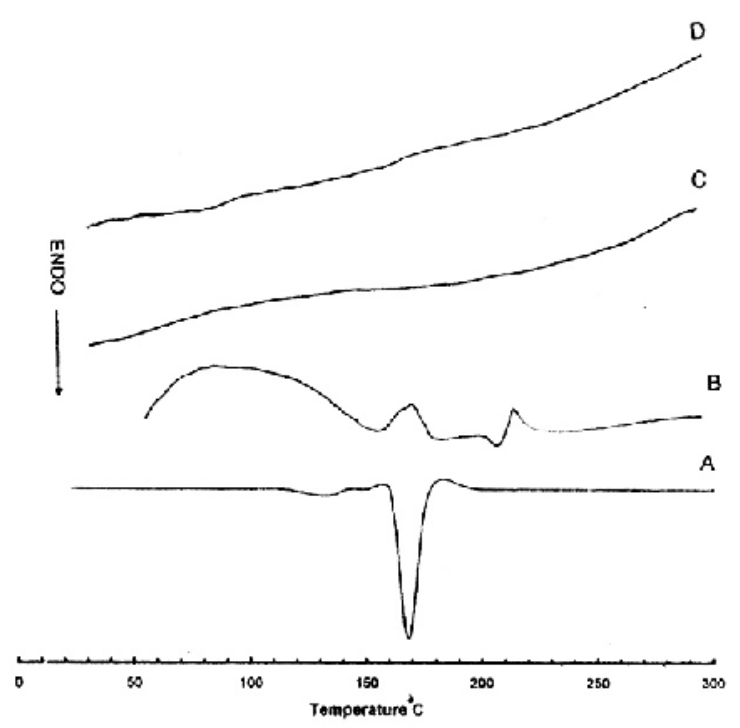

Fig. 1: DSC thermograms of dipyridamole with DM$\beta C D$ and cellulose acetate phthalate (CAP).

A- Dipyridamole alone

B- Coevaporate with DM- $\beta$ CD 1:2

C- Modified granules

D- Granules with CAP 1:3

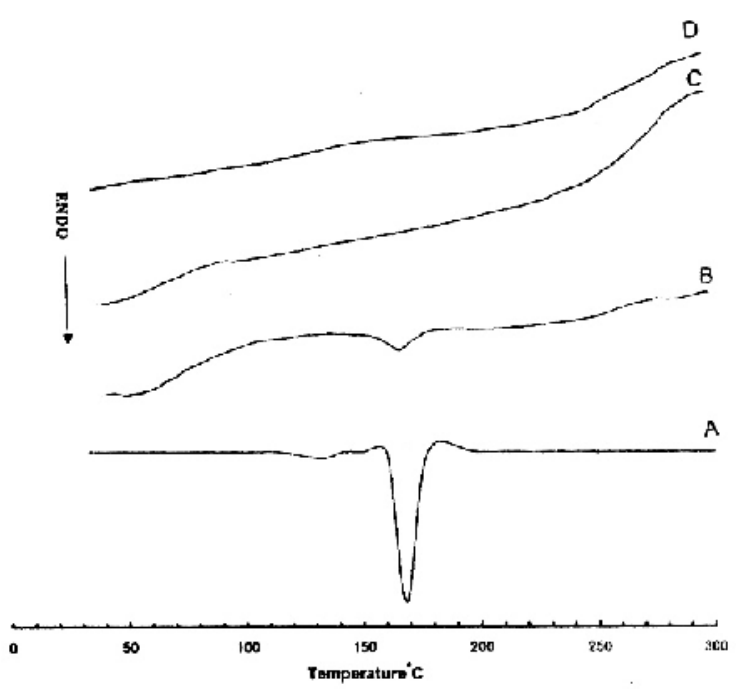

Fig. 2: DSC thermograms of dipyridamole with HP$\beta C D$ and cellulose acetate phthalate (CAP).

A- Dipyridamole alone

B- Coevaporate with HP- $\beta$ CD 1:2

C- Modified granules

D- Granules with CAP 1:3 
Powder X-ray diffraction patterns of the investigated samples

Figures (3) and (4) show powder X-ray diffraction patterns of DIP in different systems. The x-ray diffractogram of DIP crystals (curve A) had a characteristic crystalline diffraction peaks. In Fig. (3) curves B, C and D, no appearance of the characteristic diffraction peaks of DIP is recorded in case of its coevaporate, its modified granules containing $D M-\beta C D$ and CAP as well as its granules with CAP. These results, thus, revealed the formation of amorphous state of drug in these systems. Fig. (4) shows the powder x-ray diffractograms of DIP with HP- $\beta C D$ prepared systems. The diffractograms (curves B and C), of the physical

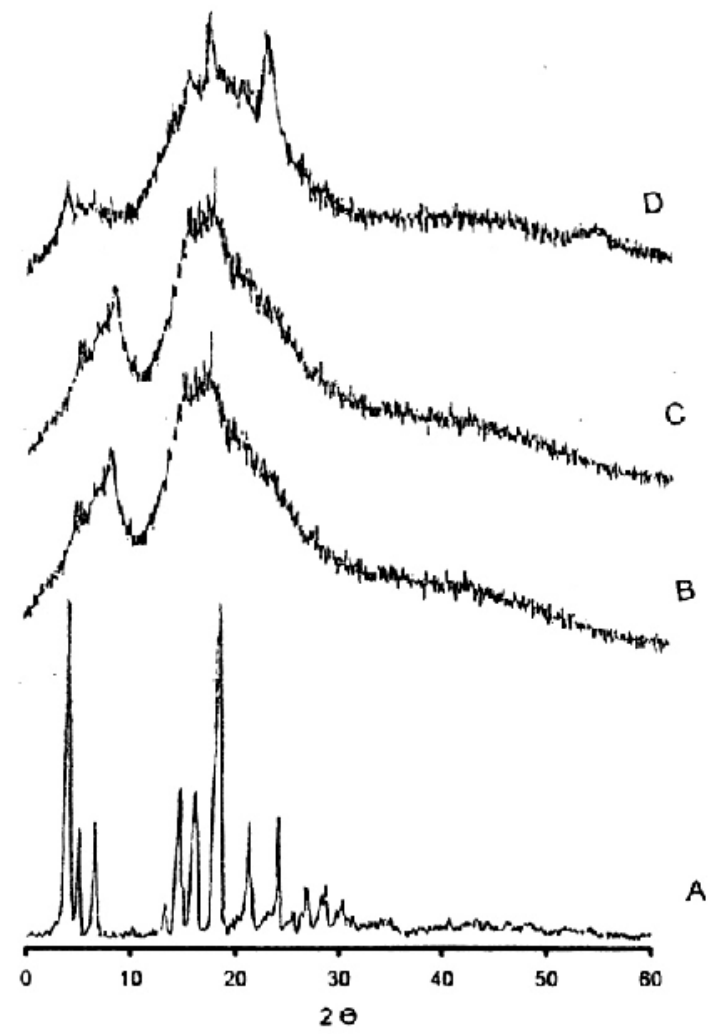

Fig. 3: X-ray diffraction patterns of dipyridamole with DM- $\beta C D$ and cellulose acetate phthalate (CAP) systems.

A- Dipyridamole alone

B- Coevaporate with DM- $\beta$ CD 1:2

C- Modified granules with DM- $\beta C D$ and CAP

D- Granules with CAP 1:3 mixture and coevaporate of DIP with HP- $\beta C D$ are similar to their crystalline patterns. This means that both physical mixture and coevaporate showed the superposition of both components; DIP and HP- $\beta C D$. On the other hand, the x-ray diffractogram of modified granules of drug with HP- $\beta C D$ coated by CAP (curve D), gave halo diffraction pattern which indicates the transformation of the drug from crystalline to amorphous state by coating. Also granules of DIP with CAP (Curve E) show a halo diffraction pattern. These x-ray diffraction data of DIP in different systems are in good agreement with the data obtained with DSC techniques of the respective samples.

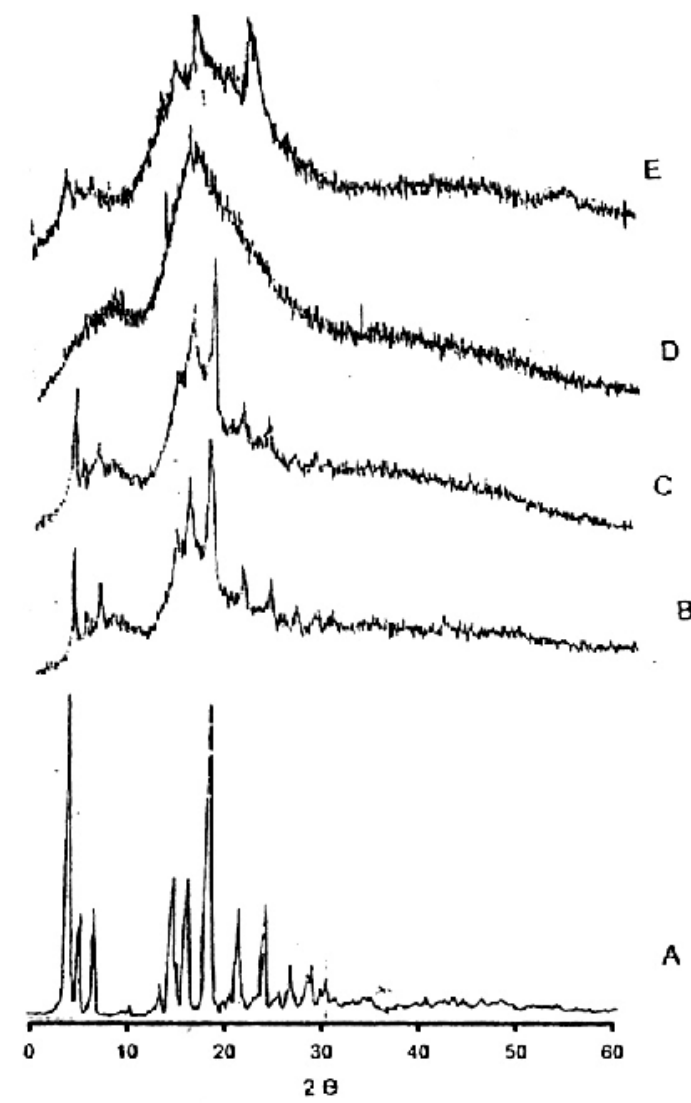

Fig. 4: X-ray diffraction patterns of dipyridamole with HP- $\beta C D$ and cellulose acetate phthalate (CAP) systems.

A- Dipyridamole alone

B- Physical mix with HP- $\beta$ CD $1: 2$

C- Coevaporate with HP- $\beta C D$

D- Modified granules with HP- $\beta C D$ and CAP

E- Granules with CAP 1:3 


\section{Release studies}

Figures (5-7) show the release profiles of the drug alone and capsules containing modified granules of DIP with DM- $\beta C D$, HP$\beta C D$ and $C A P$ at the investigated buffer solutions of $\mathrm{pH} 1.3 \& 5 \& 6.8$. At $\mathrm{pH} 1.3 \mathrm{a}$ reduction in drug release rate was achieved within $90 \mathrm{~min}$. As shown in Figure (5), the capsules containing modified granules of HP$\beta C D$ showed more retardation of DIP release than that achieved with the capsules containing modified granules of DM- $\beta C D$ in $\mathrm{pH} 1.3$. This may be attributed to the high capacity of DM$\beta C D$ to solubilize the drug. The release profiles at $\mathrm{pH} 5$ are shown in Fig. (6). It is obvious that the granules containing DM- $\beta C D$ enhanced the release of drug whereas that containing HP$\beta C D$ delayed the drug release. This may be attributed to the effect of coating with CAP which did not dissolve at $\mathrm{pH} 5$.

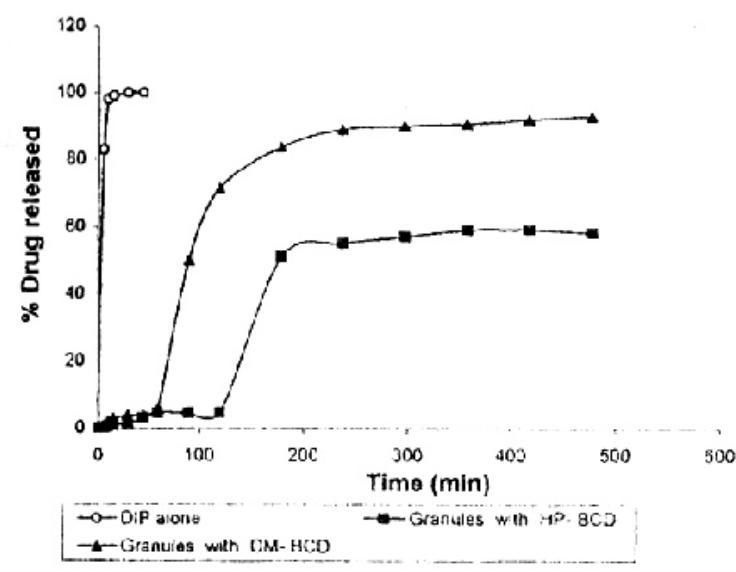

Fig. 5: Release profiles of DIP and the modified granules at $\mathrm{pH} 1.3$.

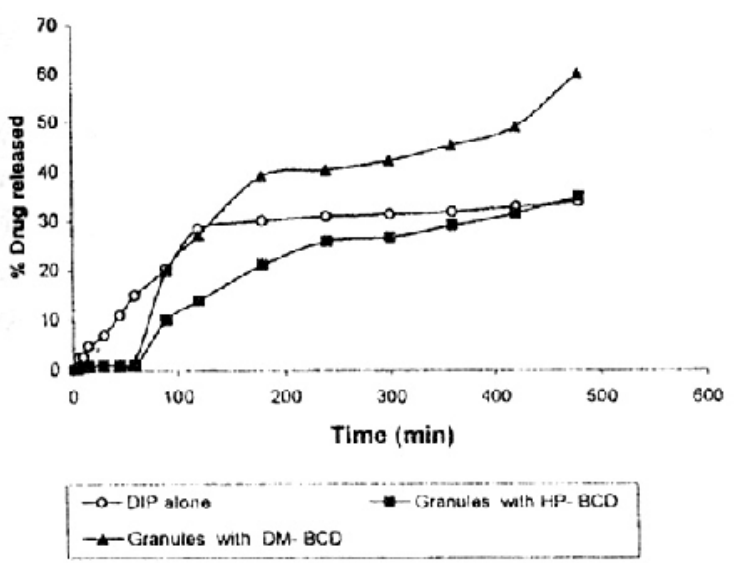

Fig. 6: Release profiles of DIP and the modified granules at $\mathrm{pH} 5$.
Figure (7) shows the release profiles of the drug alone, capsules containing modified granules of DIP with CDs and CAP, and granules without $\mathrm{CDs}$ at $\mathrm{pH}$ 6.8. It is clear that a high change in DIP release was observed, where more than $90 \%$ of DIP was released after $3 \mathrm{hrs}$ of dissolutin and about $98 \%$ of drug was released within $8 \mathrm{hrs}$ of dissoluton. This is in contrast to the release of DIP alone where not more than $20 \%$ of the drug was released within the same period. The high increase of release rate may be attributed to the desintegration of the enteric polymer of CAP by the hydrolytic effect of simulated intestinal fluids and this allowed the drug-CD complex to play its role in enhancement of DIP dissolution in the intestinal fluids. Both types of the modified granules of DIP with either DM- $\beta C D$ or HP$\beta C D$ and which are coated with CAP, showed a similar behaviour at $\mathrm{pH} 6.8$.

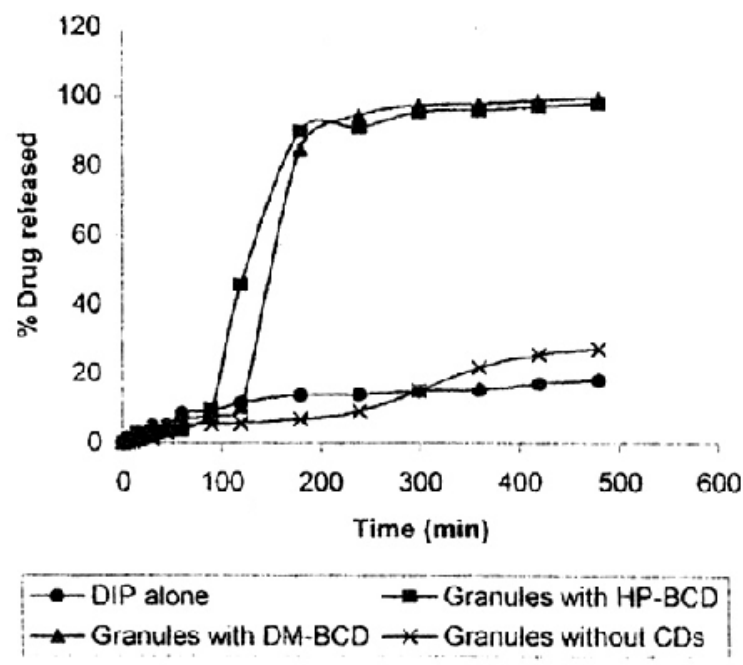

Fig. 7: Release profiles of DIP and the modified granules at $\mathrm{pH} 6.8$.

Figure (8) shows the release profiles of DIP alone and of its modified granules at the investigated $\mathrm{pHs}$, where dissolution was carried out at $\mathrm{pH} 1.3$ from 0 to less than 2 hours, at $\mathrm{pH}$ 5 from 2 hours to 5 hours and at $\mathrm{pH} 6.8$ from 5 to 8 hours. It was found that the release of the drug was highly decreased at a low $\mathrm{pH}$ based on the effect of enteric polymer (CAP) which is insoluble at acidic $\mathrm{pH}$, thus acting as a barrier against drug release at this $\mathrm{pH}$. Then, the release of drug was improved at a higher $\mathrm{pH}$ of simulated intestinal fluid, where the enteric polymer (CAP) was dissolved at $\mathrm{pH}$ above 5.5 
allowing the drug to be easily released from its complex at such a high $\mathrm{pH}$. The extent of modification of drug release from the modified granules verified the need to improve dissolution and absorption of drug through the various $\mathrm{pHs}$ of GIT.

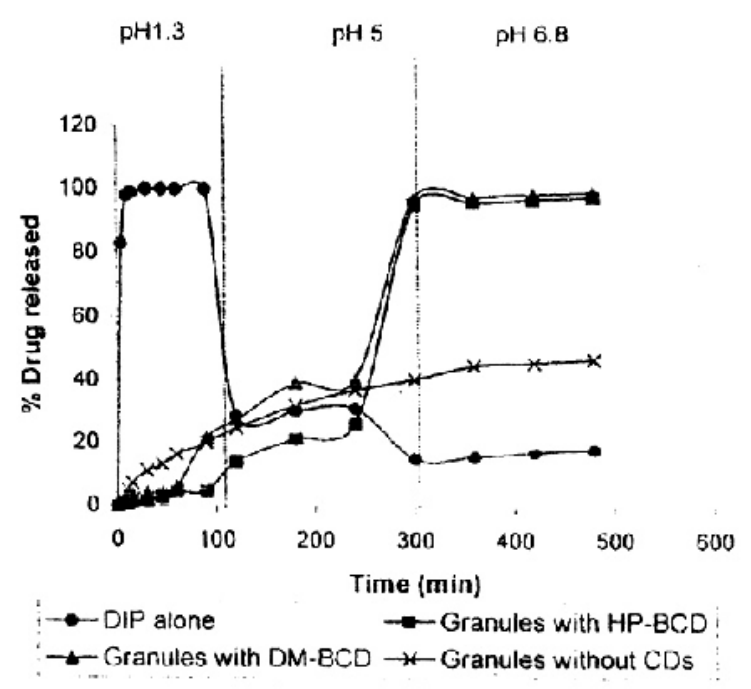

Fig. 8: Release profiles of DIP and the modified granules at $\mathrm{pH} 1.3,5$ and 6.8 .

Figure (9) shows the histogram of the release profiles for DIP and the modified granules at the investigated $\mathrm{pHs}$.

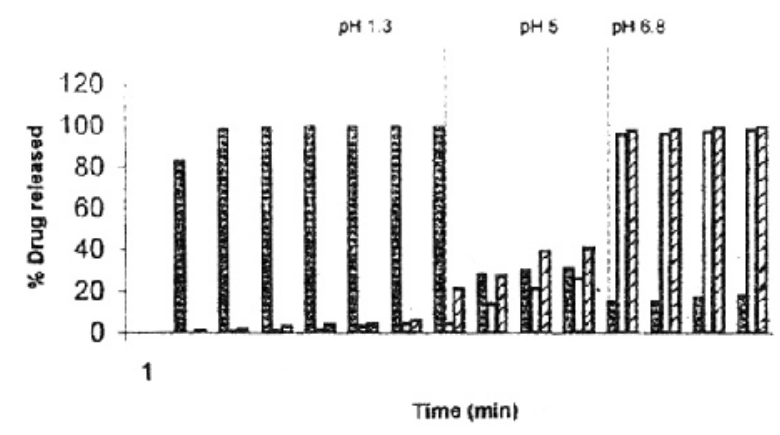

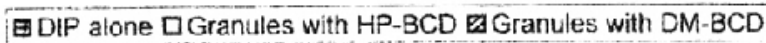

Fig. 9: Histogram of the release profiles of DIP and the modified granules at $\mathrm{pH} \mathrm{1.3,5}$ and 6.8 .

\section{Conclusions}

The behaviour of drug release from the modified granules was profoundly altered in comparison with the pure drug, by moderating the drastic dissolution behaviors of a basic drug as dipyridamole at different physiological $\mathrm{pH}$ values. The modified granules of DIP containing either HP- $\beta C D$ or DM- $\beta C D$ and coated with the enteric polymer CAP achieved more retardation of drug release at acidic $\mathrm{pH}$ of 1.3 and enhancement of drug release at intestinal $\mathrm{pH}$ of 6.8 .

\section{REFERENCES}

1- W. I. Chiou and S. Riegelman, J. Pharm. Sci., 60, 1281-1302 (1971).

2- A. Hasegawa, R. Kawamura, H. Nakagawa and I. Sugimoto, Chem. Pharm. Bull., 34, 2183-2190 (1986).

3- A. J. Moës and E. T. Recherche, J. Pharm. Belg., 44, 60-70, (1989).

4- P. Mura, A. Manderioli, G. Bramanti and L. Ceccarelli, Drug. Dev. Ind. Pharm., 22, 909 (1996).

5- S. H. Khidr, Bull. Pharm. Sci., Assuit University, 17, 81 (1994).

6- G. M. Khan and Z. Jiabi, Drug. Dev. Ind. Pharm., 24, 455 (1998).

7- P. D. Filippis, G. Zingone, M. Gibellini and P. Rupena, Eur. J. Pharm. Sci., 3, 265 (1995).

8- D. Duchene, In Cyclodextrins and Their Industrial Use, Edited by de Santa, Paris, 1987, p. 131.

9- O. Bekers, E. V. Vijtendall, J. H. Beijenen, A. Bult and W. J. M. Underberg, Drug Dev. Ind. Pharm., 17, 1503, (1991).

10- H. Uera, D. Ou, T. Enda, H. Nagase, K. Tomono and T. Najai, ibid., 24, 863, (1998).

11- T. Srini, P. Porranel, K. Ram and M. Tarun, J. Pharm. Sci., 87, 425, (1998).

12- M. M. Gamal, Master Thesis, Comparative Evaluation of Solid Dispersions and Cyclodextrin Inclusion Complexation for Clonazepam in Different Formulations, Faculty of Pharmacy, Assuit University, Assuit, Egypt (1999). 